\section{Development of L-PLA based Intrascleral Implant for Sustained Intraocular Delivery of Dexamethasone Sodium Phosphate}

\author{
Nazia Zaman ${ }^{1}$, Md. Mesbah Uddin Talukder ${ }^{1 *}$, Tasnuva Haque ${ }^{2}$, Md. \\ Khairul Alam ${ }^{1}$, Kanij Fatema ${ }^{2}$ \\ Department of Pharmacy, The University of Asia Pacific ${ }^{1}$ \\ Dhaka-1209, Bangladesh \\ Department of Pharmacy, Stamford University Bangladesh ${ }^{2}$ \\ 51, Siddeswari Road, Dhaka-1217, Bangladesh
}

${ }^{*}$ Corresponding Author

Md. Mesbah Uddin Talukder Lecturer, Dept. of Pharmacy, The University of Asia Pacific, Contact no.: +8801712143717 E-mail: mesbah777@yahoo.com

\begin{abstract}
The present study was carried out to develop biodegradable intrascleral implants of Dexamethasone Sodium Phosphate and to evaluate the release pattern of the drug from the prepared implants. Intrascleral implants were prepared by using biodegradable polymer L-PLA (m.wt. 61,200 Da). Sodium chloride ( $\mathrm{NaCl})$, gelatin and glycerol monostearate (GMS) were used in various formulations to observe the effects of these additives on the release of Dexamethasone Sodium Phosphate from the prepared L-PLA based intrascleral implants. Five different formulations were prepared for this study and were coded as FD-1 (10\%drug+L-PLA), FD-2 (20\%drug+L-PLA), FD-3 (10\%drug+L-PLA+5\%NaCl), FD-4 (10\%drug+L-PLA $+5 \%$ Gelatin) and FD-5 (10\%drug+L-PLA+10\% GMS). Discs were prepared and made into appropriate shape before submerging into the buffer solution of $\mathrm{pH} 7.4$ in different vials. The in vitro release profile of Dexamethasone Sodium Phosphate from the implants showed a biphasic release pattern with an initial burst followed by a diffusive phase. It was observed that FD-1 and FD-2 showed $19.63 \%$ and $29.87 \%$ release on the first day and $24.22 \%$ and $38.5 \%$ release respectively at day 30 . The drug loading of FD-1 and FD-2 was $10 \%$ and $20 \%$ respectively. Among FD-3, FD-4 and FD-5; FD-3 showed highest release (32.1\%) at day 30 in which 5\% $\mathrm{NaCl}$ was used. FD-4 showed $27.45 \%$ release at day 30 where gelatin, a hydrophilic agent was used and FD5 containing GMS, a lipid material, was found to be most retarding (19.22\% at day 30$)$. The results of the dissolution study provide an idea that L-PLA may be successfully used for the preparation of biodegradable intrascleral implant of Dexamethasone Sodium Phosphate.
\end{abstract}

Key words: Dexamethasone Sodium Phosphate, Bioidegradable polymer, Intrascleral implants.

\title{
INTRODUCTION
}

Eye is a sensitive organ and provides the sense of sight but yet it is one of the most inaccessible organs because of its layers and textures. Oral administration of drugs has proven to be very slow in action and topical administration such as creams and ointments though is effective, fails to be uniform in dosage and to achieve patient compliance. It has been hypothesized that trans-scleral delivery is an effective method of achieving therapeutic concentrations of drugs in the posterior part of the eye (Okabe et.al., 2003). With its large surface area and high degree of hydration, the sclera is permeable to solutes of a wide range of molecular weights (Ambati et al., 2000). Intraocular drug delivery systems are being investigated to maintain long-term vitreous concentration of drugs in the therapeutic range. Although intravitreal injection of particulate systems such as biodegradable microspheres (Moritera et al., 1991) and liposomes (Peyman et al., 1987) may be useful for the treatment of vitreoretinal disorders but small particles suspended in the vitreous might impair the clarity of the ocular medium. Trans-scleral iontophoresis has been suggested as a potentially useful noninvasive technique for intravitreal delivery of ionizable drugs (Barza et al., 1986) but it requires complicated procedures and its application is generally limited to ionic drugs. There are two main advantages for using an intraocular perfusion system (Michelson et al., 1979) or intraocular microdialysis (Waga et al., 1995): they can administer the drug into the vitreous cavity continuously, and administration of the drug can be changed or stopped at any time. However, permanent access to the intraocular space may increase the risk of intraocular infection. Many intraocular inflammatory and proliferative diseases such as uveitis, diabetic retinopathy and proliferative vitreoretinopathy, require long-term treatment with the drug (Okabe 
et.al., 2003). In this study, a biodegradable intrascleral implant containing Dexamethasone Sodium Phosphate was used to evaluate its feasibility as a sustained intraocular drug delivery system. Here the implants were prepared by a new technique. The percent potency and the in vitro release rate of drug were also investigated.

\section{EXPERIMENTAL}

\section{Materials}

Dexamethasone Sodium Phosphate used in this experiment was a gift sample of Opsonin Pharmaceuticals Ltd. L-PLA (molecular wt. 61,200 Da) was collected from DURECT Corporation (Lactel Polymer). Glycerol monostearate (GMS), sodium chloride $(\mathrm{NaCl})$ and gelatin were from Merck KGaA (Germany), Thomas Baker (India) and Loba Chemie (India) respectively. A hydraulic press of Perkin- Elmer (U.S.A), a UV-vis Spectrophotometer of Shimadzu (Japan), a shaking water bath of Memmert (Germany), an electronic balance of Shimadzu (Japan) and a pH meter of Hanna (U.S.A) were used.

\section{Preparation of implants with Dexamethasone Sodium Phosphate}

The drug, polymer and excipients were weighed out accurately in an electronic balance according to the formulations given in Table 1. Those formulation ingredients were mixed homogenously using a sophisticated mortar and pestle. Appropriate amount of the mixture was taken in a PerkinElmer hydraulic press equipped with $13 \mathrm{~mm}$ diameter flat-faced punch previously lubricated with $1 \%$ dispersion of magnesium stearate in ethanol. Then 8 ton pressure was applied for 5 minutes and a circular sheet was produced which was $13 \mathrm{~mm}$ in diameter. Smaller discs of $4 \mathrm{~mm}$ diameter were cut out using a sharp cork borer (Figure 1). Finally, the discs were kept in desiccators.

\section{Preparation of $\mathrm{pH}$ 7.4 Phosphate buffer}

In a $1000 \mathrm{ml}$ volumetric flask, $405 \mathrm{ml} 0.2 \mathrm{M}$ disodium hydrogen phosphate $\left(\mathrm{Na}_{2} \mathrm{HPO}_{4}\right)$ and $95 \mathrm{ml}$ $0.2 \mathrm{M}$ sodium dihydrogen phosphate $\left(\mathrm{NaH}_{2} \mathrm{PO}_{4}\right)$ were taken and distilled water was added upto 1 litre. The $\mathrm{pH}$ of the prepared solution was adjusted to 7.4 using Hanna $\mathrm{pH}$ meter.

\section{Potency determination}

One intrascleral disc of each formulation was crushed in a mortar using a pestle. A certain amount $(10 \mathrm{mg})$ of the crushed material was taken in $100 \mathrm{ml}$. of volumetric flask and dissolved properly by $\mathrm{pH} 7.4$ phosphate buffer. The drug content in each implant was determined by spectrophotometrically.

\section{In-vitro dissolution studies}

The intrascleral implant was placed in $2 \mathrm{ml}$ of dissolution medium ( $\mathrm{pH} 7.4$ phosphate buffer) in a closed vial. Then it was immersed in a shaking water bath at $37^{\circ} \mathrm{C}$. At 10 days intervals, the entire volume was sampled and $2 \mathrm{ml}$ fresh medium was added to the sample vial to approximate a perfect sinking condition (Okabe et al., 2003). The release of Dexamethasone Sodium Phosphate was determined measuring the absorbance using UV-vis Spectrophotometer at $243 \mathrm{~nm}$.

The mechanism of release pattern of Dexamethasone Sodium Phosphate was explained using Korsmeyer-peppas equation (Korsmeyer et al., 1983):

$\mathrm{M}_{\mathrm{t}} / \mathrm{M}_{\alpha}=\mathrm{Kt}^{\mathrm{n}}$.....

Where $M_{t} / M_{\infty}$ is the fractional solute release, $t$ is the release time, $K$ is a kinetic constant characteristic of the drug/polymer system and $\mathrm{n}$ is an exponent that characterizes the mechanism of release of drug. For cylindrical matrix tablets, if the exponent $n=0.45$, then the drug release mechanism is Fickian diffusion and if $0.45<n<0.89$, then it is non-Fickian or anomalous diffusion. An exponent value of 0.89 is indicative of Case-II transport or typical zero-order release (Siepmann et al., 2001).

\section{RESUTLS AND DISCUSSION}

\section{Assay result}

According to the procedure stated above, the percent potency of the intrascleral discs of all formulations was found within 99-101. 
Table 1: Different formulations for preparing Dexamethasone Sodium Phosphate loaded intrascleral implants.

\begin{tabular}{|c|c|c|c|c|c|}
\hline \multirow{2}{*}{ Formulations } & Drug & Polymer & \multicolumn{3}{|c|}{ Excipients } \\
\cline { 2 - 6 } & Dexamethasone (mg) & $\begin{array}{c}\text { L-PLA (61, } \\
\text { 200) (mg) }\end{array}$ & $\begin{array}{c}\text { Gelatin } \\
\text { (mg) }\end{array}$ & $\begin{array}{c}\text { NaCl } \\
\text { (mg) }\end{array}$ & $\begin{array}{c}\text { GMS } \\
\text { (mg) }\end{array}$ \\
\hline FD-1 & 22 & 198 & - & - & - \\
\hline FD-2 & 44 & 176 & - & - & - \\
\hline FD-3 & 22 & 187 & - & 11 & - \\
\hline FD4 & 22 & 187 & 11 & - & - \\
\hline FD-5 & 22 & 176 & - & - & 22 \\
\hline
\end{tabular}

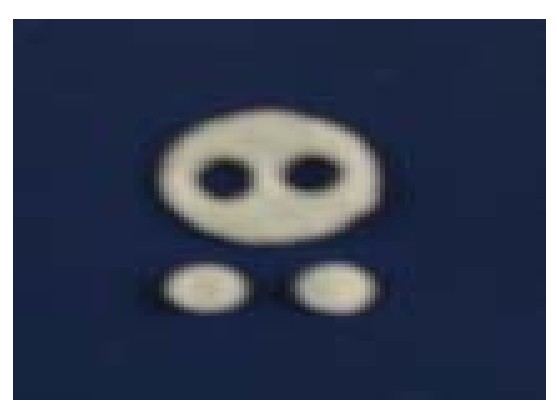

Figure 1: Circular sheet of $13 \mathrm{~mm}$ diameter was cut out to produce implants of $4 \mathrm{~mm}$ diameter.

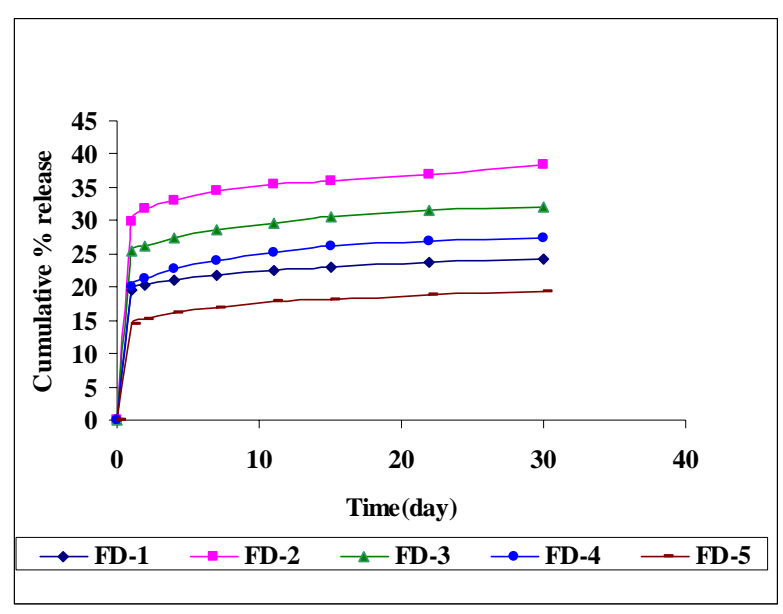

Figure 2: Biphasic release profile of Dexamethasone Sodium Phosphate from different formulations of intrascleral implants.

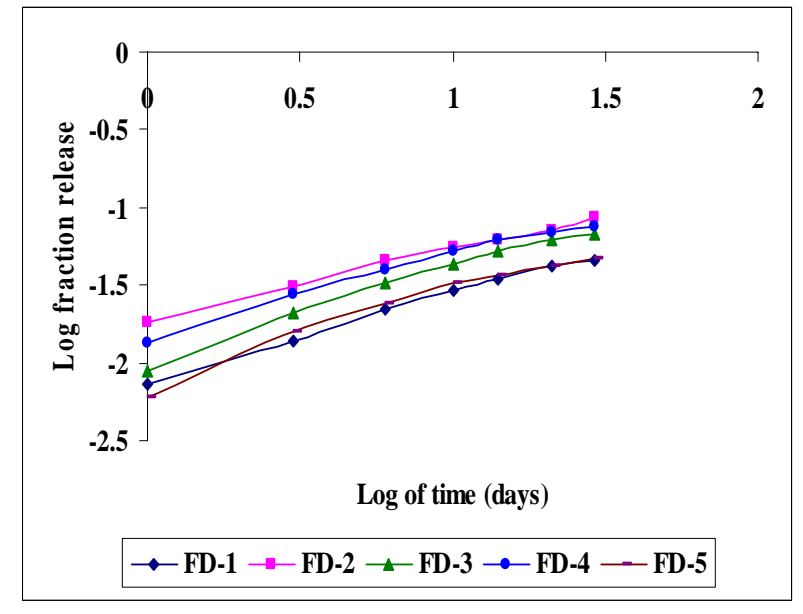

Figure 3: Release profile of Dexamethasone Sodium Phosphate from different formulations of intrascleral implants (Korsmeyer plot). 
Table 2: Interpretation of regression coefficient $\left(R^{2}\right)$ value and release exponent $(n)$ from Korsmeyer plot.

\begin{tabular}{|c|c|c|}
\hline Formula & $\mathbf{n}$ & $\mathbf{R}^{\mathbf{2}}$ \\
\hline FD-1 & 0.5589 & 0.9930 \\
\hline FD-2 & 0.4520 & 0.9926 \\
\hline FD-3 & 0.6072 & 0.9832 \\
\hline FD-4 & 0.5169 & 0.9830 \\
\hline FD-5 & 0.6039 & 0.9662 \\
\hline
\end{tabular}

\section{Dissolution studies}

In this study, the feasibility of using a biodegradable polymer, L-PLA as an intraocular sustained release system for Dexamethasone Sodium Phosphate was demostrated. Moreover, the effect of drug load and different excipients (gelatin, GMS and $\mathrm{NaCl}$ ) on L-PLA based intrascleral implants were observed.

The in vitro release profile of Dexamethasone Sodium Phosphate from the implant showed a biphasic release pattern (Figure 2), with an initial burst followed by a diffusive phase. This release profile was similar to that of a biodegradable plug-shaped scleral implant containing Betamethasone Sodium Phosphate, which was reported earlier (Kunou et al., 2000). The initial burst may have been due to the rapid release of the drug deposited on the implant surface and in the water channels in the matrix. The second release phase showed slow drug release, possibly controlled by the degradation rate of the polymer. During the next diffusive phase, the implant continued releasing Dexamethasone Sodium Phosphate for a long period of time. The dissolution data of 30 days were taken into consideration for analysis.

It was observed that FD-1 and FD-2 showed $19.63 \%$ and $29.87 \%$ release on first day and $24.22 \%$ and $38.5 \%$ release respectively at day 30 . The drug loading of FD-1 and FD-2 was $10 \%$ and $20 \%$ respectively. So, it could be stated that increased drug loading enhanced the release of drug from the implants. Among FD-3, FD-4 and FD-5, FD-3 showed highest release (32.1\%) at day 30 as in that formulation $5 \% \mathrm{NaCl}$ was used. The observed lowest rate retarding action of L-PLA was attributed to the channeling effect of $\mathrm{NaCl}$ which was found similar to that of Razzak et al., 2008. FD-4 showed $27.45 \%$ release at day 30 in which gelatin, a hydrophilic agent was used that enhanced the release rate of drug from L-PLA. FD-5 containing GMS, was found to be most retarding (19.22\% at day 30 ), probably because of the fact that GMS, itself a lipid material, showed greater extent of rate retarding action in combination with L-PLA.

To identify the mechanism of drug release in the diffusive phase from the intrascleral implants, the data of diffusive phase were treated with Korsmeyer model (Figure 3 ) and found that the data were fitted $\left(R^{2}\right.$ values were found within 0.9830 to 0.9662$)$. So, Korsmeyer model was used to explain the mechanism of drug release in the diffusive phase. It was observed that the $\mathrm{n}$ values were within 0.4520 to 0.6072 (Table 2) suggesting probable mechanism of drug release in the diffusive phase from the intrascleral implants was anomalous transport. The time to time erosion of polymer layer into aggregates or granules which in turn disaggregate into fine particles release their drug content by dissolution. The second release phase showed slow drug release, possibly mainly controlled by the degradation speed of the polymer.

\section{CONCLUSION}

From the above discussion, it is clear that biodegradable intrascleral implants can be easily prepared by using L-PLA (mol. wt. 61,200) which may provide a long term delivery of Dexamethasone Sodium Phosphate inside the eye. Further in vivo study is required to establish the dosage form.

\section{ACKNOWLEDGEMENT}

The authors are thankful to Prof. Dr. Reza-ul Jalil, Department of Pharmaceutical Technology, University of Dhaka. Authors are also thankful to Opsonin Pharmaceuticals Ltd. for providing gift samples of Dexamethasone Sodium Phosphate. 


\section{REFERENCES}

Ambati J, Canakis CS, Miller JW, Gragoudas ES, Edwards A, Weissgold DJ, Kim I, Delori FC, Adamis AP. (2000) Diffusion of high molecular weight compounds through sclera. Investigative Ophthalmology \& Visual Science. 41(5): 1181-1185.

Barza M, Peckman C, Baum J. (1986) Transscleral iontophoresis of cefazolin, ticarcillin and gentamicin in the rabbit. Ophthalmology. 93: 133-139.

Korsmeyer RW, Gurny R, Doelker E, Buri P, Peppas NA. (1983) Mechanisms of solute release from porous hydrophilic polymers. Int. J. Pharm. 15: 25-35.

Kunou N, Ogura Y, Honda Y, Hyon SH, Ikada Y. (2000) Biodegradable scleral implant for controlled intraocular delivery of betamethasone phosphate. J. Biomed. Mater. Res. 51: 635-641.

Michelson JB, Nozik RA. (1979) Experimental endophthalmitis treated with an implantable osmotic minipump. Arch Ophthalmol. 97: 1345-1346.

Moritera T, Ogura Y, Honda Y, Wada R, Hyon SH, Ikada Y. (1991) Microspheres of biodegradable polymers as a drug-delivery system in the vitreous. Invest. Ophthalmol. Vis. Sci. 32: 17851790.

Moritera T, Ogura Y, Yoshimura N, Honda Y, Wada R, Hyon SH, Ikada Y. (1992). Biodegradable microspheres containing adriamycin in the treatment of proliferative vitreoretinopathy. Invest. Ophthalmol. Vi.s Sci.33: 3125-3130.

Okabe J, Kimura H, Kunou N, Okabe K, Kato A, Ogura Y. (2003) Biodegradable intrascleral implant for sustained intraocular delivery of betamethasone phosphate. Investigative Ophthalmology \& Visual Science. 44(2): 740-744.

Peyman GA, Khoobehi B, Tawakol M, Schulman JA, Mortada HA, Alkan H, Fiscella R. (1987) Intravitral injection of liposome encapsulated ganciclovir in a rabbit model. Retina. 7: 227229.

Razzak MSMI, Khan F, Khan MZR, Fatema K, Islam MS, Reza MS. (2008) Effect of channeling agents on the release profile of theophylline from METHOCEL K4M based matrix tablets. Dhaka Univ. J. Pharm. Sci. 7(1): 27-32.

Siepmann J, Peppas NA. (2001) Modeling of drug release from delivery systems based on hydroxypropyl methylcellulose (HPMC). Adv. Drug Deliv. Rev. 48: 139-157.

Waga J, Ehinger B. (1995) Passage of drugs through different intraocular microdialysis membranes. Grafe Arch. Clin. Exp. Ophthalmol. 233: 31-37. 